\title{
Efeitos de 5 Anos de Intervençấo Comportamental Intensiva no Desenvolvimento de uma CrianÇa Com Autismo ${ }^{1}$ \\ EFFECTS OF 5 YEARS OF INTENSIVE BEHAVIORAL INTERVENTION ON THE DEVELOPMENT OF A CHILD WITH AUTISM
}

\author{
Antônio César Gontijo Silva Assunção Montezuma ANDALÉCIO² \\ Camila Graciella Santos GOMES 3 \\ Analice Dutra SILVEIRA ${ }^{4}$ \\ Ianaiara Marprates OLIVEIRA ${ }^{5}$ \\ Robson Cardinali CASTRO ${ }^{6}$
}

\begin{abstract}
RESUMO: O objetivo deste estudo de caso único é descrever e avaliar a aplicação de um modelo de Intervenção Comportamental Intensiva, realizado por meio da capacitação dos cuidadores, com uma criança com autismo gravemente comprometida e não falante. A intervenção ocorreu por 40 horas semanais em ambiente domiciliar e escolar, ao longo de cinco anos consecutivos. Instrumentos padronizados foram utilizados para medir o desenvolvimento da criança. Os resultados gerais indicaram ganhos no desenvolvimento da criança e a viabilidade da capacitaçáa dos cuidadores para esse tipo de intervençáo.
\end{abstract}

PALAVRAS-CHAVE: Autismo. Educaçăo Especial. Cuidadores. Análise do Comportamento Aplicada. Intervenção intensiva.

ABSTRACT: The purpose of this single case study is to describe and evaluate the application of an Intensive Behavioral Intervention model, carried out through the training of caregivers, with a child with severely compromised and non-speaking autism. The intervention occurred for 40 hours a week in a home and school environment, during five consecutive years. Standardized instruments were used to measure child development. Overall results indicated gains in child development and the feasibility of training of caregivers for this type of intervention.

KEYWORDS: Autism. Special Education. Caregivers. Applied Behavior Analysis. Intensive intervention.

\section{INTRODUÇÃo}

Intervençóes Comportamentais Intensivas têm demonstrado efeitos significativos no desenvolvimento de pessoas com autismo, desde a década de 1980 (Boyd \& Corley, 2001; Campbell, Schopler, Cueva, \& Hallin, 1996; Dawson et al., 2010; Lovaas, 1987; Smith, 1999; Warren et al., 2011). Esse tipo de intervenção é caracterizada pela estimulação individualizada

\footnotetext{
${ }^{1}$ http://dx.doi.org/10.1590/s1413-65382519000300003

${ }^{2}$ Graduando em Psicologia pela Faculdade de Ciências Médicas de Minas Gerais. acgsama@gmail.com. Belo Horizonte/Minas Gerais/ Brasil. ORCID: https://orcid.org/0000-0002-6229-4417

${ }^{3}$ Doutora em Educação Especial pela UFSCar, diretora do CEI Desenvolvimento Humano, docente da Faculdade de Ciências Médicas de Minas Gerais e membro do Instituto Nacional de Ciência e Tecnologia sobre Comportamento, Cogniçẫo e Ensino (FAPESP: 2014/50909-8; CNPq: 465686/2014-1; CAPES: 88887.136407/2017-00). camilagsg@gmail.com. Belo Horizonte/ Minas Gerais/ Brasil. ORCID: https://orcid.org/0000-0002-4268-328X

${ }^{4}$ Mestra em Educação Especial pela UFSCar e diretora do CEI Desenvolvimento Humano. analicesilveira2013@gmail.com. Belo Horizonte/Minas Gerais/ Brasil. ORCID: https://orcid.org/0000-0002-2299-4443

${ }^{5}$ Psicóloga do CEI Desenvolvimento Humano. ianaiaraprates@gmail.com. Belo Horizonte/Minas Gerais/ Brasil . ORCID: https://orcid.org/0000-0001-5603-2173

${ }^{6}$ Psicólogo do CEI Desenvolvimento Humano. robsoncardin@gmail.com. Belo Horizonte/Minas Gerais/ Brasil. ORCID: https://orcid.org/0000-0003-1010-2978
} 
(um educador para uma criança com autismo), realizada por muitas horas semanais (de 15 a 40 horas), por pelo menos dois anos consecutivos, que abrange várias áreas do desenvolvimento simultaneamente e que é fundamentada em princípios de Análise do Comportamento (Green, 1996), definida como Análise do Comportamento Aplicada (Applied Behavior Analysis [ABA]; Baer, Wolf, \& Risley, 1987).

O primeiro estudo sobre Intervenção Comportamental Intensiva aplicada ao tratamento do autismo foi publicado por Lovaas (1987) e, após essa publicação, dezenas de estudos posteriores replicaram parcialmente os resultados do estudo inicial (Smith, Buch, \& Gamby, 2000; Warren et al., 2011), os quais utilizaram educadores diferentes (estudantes, profissionais e familiares) e contextos múltiplos (instituiçóes especializadas, escolas e a residência das crianças). As pesquisas indicaram, no geral, melhora no desenvolvimento dos participantes após realizarem esse tipo de intervenção. Esses estudos descreveram resultados variados em relação à proporção dos ganhos da intervenção, mas, de modo geral, indicaram ganhos no desenvolvimento das crianças, especialmente quando realizada precocemente (Smith et al., 2000; Warren et al., 2011).

No Brasil, dois estudos descreveram os efeitos de um modelo de Intervenção Comportamental Intensiva aplicado ao tratamento de crianças brasileiras com autismo. Gomes et al. (2019) avaliaram os efeitos desse tipo de intervenção, realizada por meio da capacitação de cuidadores, no desenvolvimento de crianças com autismo e compararam as crianças que realizaram a intervenção com crianças que não realizaram. Os participantes foram atribuídos a dois grupos: o Grupo 1 era composto por 22 crianças que finalizaram o primeiro ano de intervenção intensiva, e o Grupo 2 era composto por 11 crianças que não fizeram a intervenção intensiva. Os resultados indicaram ganhos significativos em todas as áreas do desenvolvimento das crianças com autismo que passaram pelo primeiro ano de Intervenção Comportamental Intensiva, enquanto as crianças do grupo sem intervenção intensiva apresentaram ganhos menos expressivos. Crianças mais novas, que falavam e que apresentavam sintomas mais brandos de autismo, obtiveram resultados melhores.

Em outro estudo, Gomes, de Souza, Silveira e Oliveira (2017) avaliaram os efeitos do primeiro ano de Intervenção Comportamental Intensiva no desenvolvimento de nove crianças com autismo, com idades entre 1 ano e 3 meses e 2 anos e 11 meses, atendidas por um centro brasileiro especializado. A intervenção, conduzida por aproximadamente 15 horas semanais, foi realizada na residência dos participantes por meio da capacitação dos cuidadores. As crianças foram avaliadas no início e ao término da intervençáo. Os dados foram analisados individualmente, e os resultados indicaram ganhos no desenvolvimento.

O objetivo deste estudo de caso único foi verificar os efeitos da aplicação de um modelo de Intervenção Comportamental Intensiva, realizado por meio da capacitação dos cuidadores, no desenvolvimento de uma criança com autismo gravemente comprometida e não falante. A intervenção ocorreu por 40 horas semanais em ambiente domiciliar e escolar, ao longo de cinco anos consecutivos. Dois instrumentos padronizados foram utilizados anualmente para medir o desenvolvimento da criança, permitindo avaliar possíveis ganhos decorrentes da intervenção. 


\section{Método}

Nesta seção, serão apresentados o participante desta pesquisa, os instrumentos e os procedimentos de avaliação, a intervenção, os procedimentos de ensino das habilidades trabalhadas em cada programa e os éticos.

\subsection{Participante}

Participou do estudo uma criança de 2 anos e 2 meses, não falante, com diagnóstico prévio de autismo, sem o diagnóstico de comorbidades, realizado por psiquiatra infantil especializado, seguindo os critérios da Classificação Internacional de Doenças - CID 10 (Organização Mundial de Saúde [OMS], 1993). A criança foi acompanhada pelo Centro de Estudos e Intervenção para o Desenvolvimento Humano (CEI) entre maio de 2012 e maio de 2017, totalizando 5 anos de intervenção.

\subsection{InSTRUMENTOS DE AVALIAÇÁO}

Para avaliar o desenvolvimento da criança, foram utilizados o Psychoeducational Profile-Revised-PEP-R (Schopler, Reichler, Bashford, Lansing, \& Marcus, 1990) e o Inventário Portage Operacionalizado - IPO (Williams \& Aiello, 2001). Optou-se pela utilização desses dois instrumentos porque ambos foram adaptados para a população brasileira; medem, em anos e meses, áreas importantes do desenvolvimento isoladamente, mas também oferecem uma medida global do desenvolvimento, o que permite comparar a criança avaliada com o que é esperado para a idade cronológica; permitem avaliar os efeitos da intervenção no desenvolvimento da criança, em áreas específicas e o desenvolvimento como um todo.

O PEP-R avalia tanto o atraso no desenvolvimento como comportamentos típicos de autismo e oferece informaçóes sobre sete áreas na Escala de Desenvolvimento: imitação, percepção, coordenação motora fina, coordenação motora grossa, integração olho-mão, desenvolvimento cognitivo e cognitivo verbal, além de quatro áreas na Escala de Comportamento: linguagem, relacionamento e afeto, respostas sensoriais e interesses por materiais. Pode ser utilizado com crianças de 6 meses a 12 anos de idade. Foi adaptado e validado para a população brasileira (Leon, Bosa, Hugo, \& Hutz, 2004). Neste estudo, foram utilizados os dados da Escala de Desenvolvimento (não os da Escala de Comportamento).

O IPO avalia o padrão de desenvolvimento infantil em cinco áreas: linguagem, socialização, desenvolvimento motor, cognição e autocuidados, em períodos de idade que vão de 0 a 6 anos. Não é um instrumento destinado a avaliar especificamente o desenvolvimento de crianças com autismo, mas avalia o desenvolvimento de qualquer criança, independentemente do diagnóstico. O inventário foi adaptado e operacionalizado para a população brasileira (Williams \& Aiello, 2001).

Além do PEP-R e do IPO, utilizou-se também a Childhood Autism Rating Scale CARS (Schopler, Reichler \& Renner,1988), que permite identificar crianças com características comportamentais de autismo e distinguir entre autismo e atraso no desenvolvimento sem autismo. De acordo com a escala, os resultados da avaliação podem ser distribuídos em três categorias: desenvolvimento normal (15-29,5), autismo leve/moderado (30-36,5) e autismo 
grave (acima de 37). Pode ser utilizada com crianças acima de 36 meses de idade. Essa escala foi adaptada e validada para a população brasileira por Pereira, Riesgo e Wagner (2008).

\subsection{Procedimentos de aValiaçáo}

Anualmente, a criança era avaliada por dois profissionais do CEI, um com formação em Psicologia e o outro em Terapia Ocupacional, por meio da aplicação do PEP-R, IPO e CARS (considerando as idades mínimas e máximas dos instrumentos). A aplicação do PEP-R ocorria em uma sala com poucos estímulos, em situação na qual um terapeuta apresentava as atividades e o outro registrava o desempenho da criança. O IPO era aplicado na residência da criança, em situação mais próxima do natural, a partir da observação do comportamento da criança e do relato dos cuidadores. É importante ressaltar que algumas habilidades eram medidas pelos dois instrumentos, porém de maneiras distintas e com critérios diferentes.

A aplicação do PEP-R era feita em ambiente artificial (sala com poucos estímulos) e sob demanda (o avaliador solicitava que a criança fizesse a atividade); a aplicação do IPO era realizada em ambiente natural (residência do participante) por meio da observação da criança e do relato dos cuidadores, sem demandas direcionadas à criança. A CARS era preenchida pelos avaliadores com base na observação do comportamento da criança. Eram necessárias, em média, duas semanas para a aplicação dos instrumentos de avaliação, análise dos dados e redação de um relatório com os resultados.

\subsection{INTERVENÇÁO}

A intervenção ocorria na residência da criança com autismo e na escola que a criança frequentava, por meio da capacitação dos cuidadores (pais, babá e estagiários de Psicologia), realizada por profissionais do CEI; nesse sentido, quem executava as atividades com a criança eram os cuidadores, seguindo as orientações ministradas pelos profissionais do CEI.

Na residência, a criança era acompanhada pelos pais, pela babá e por um estagiário de graduação em Psicologia. O estagiário fazia as atividades diárias com a criança por 2 horas, de segunda a sexta, totalizando 10 horas semanais de estimulação, e era responsável pela realização dos programas de ensino de habilidades básicas, de fala e acadêmicas (Gomes \& Silveira, 2016; Gomes, 2015), que serão descritos a seguir. Os pais e a babá eram responsáveis pelo ensino das habilidades de autocuidados (Silveira \& Oliveira, 2018; Silveira \& Gomes, 2019), em contexto natural, e pela manutenção e pela generalização das habilidades aprendidas nas atividades realizadas com o estagiário.

Dois profissionais, um com formação em Psicologia e o outro em Terapia Ocupacional, estavam presentes na casa da criança, duas vezes por semana (cada profissional uma vez por semana), em sessões de uma hora cada. O profissional de Psicologia orientava o estagiário, os pais e a babá para o ensino das habilidades básicas, de fala e acadêmicas; o de Terapia Ocupacional orientava os pais e a babá para o ensino das habilidades de autocuidados. A função dos profissionais era ensinar os cuidadores a realizar as atividades com a criança com autismo no ambiente no qual essas habilidades seriam ensinadas (não havia um treino teórico para os cuidadores, apenas o ensino contextual). Além disso, os profissionais também ensinavam os cuidadores a 
fazer registros sistemáticos do desempenho da criança nas atividades (em protocolos elaborados pelo CEI, específicos para cada atividade; ver Gomes \& Silveira, 2016) e supervisionavam a realização correta das atividades e dos registros para garantir a fidedignidade da intervenção.

A criança frequentava uma escola regular privada, 5 vezes por semana, 4 horas por dia, totalizando 20 horas de estimulação semanal, e era acompanhada em período integral por um mediador (estagiário de Psicologia). Um profissional do CEI com formação em Psicologia frequentava a escola da criança, uma vez por semana, e orientava sistematicamente o mediador, em sessóes com duração de uma hora cada. As orientações na escola abordavam tanto as condiçóes de ensino (a preparação do ambiente e dos materiais, como apresentar as instruçóes, a exigência de resposta ativa da criança, a apresentação de consequências identificadas como potencialmente reforçadoras, entre outros aspectos), como o manejo geral dos comportamentos da criança. $\mathrm{O}$ mediador também fazia registros sistemáticos das atividades realizadas pela criança no ambiente escolar (Gonzaga \& Borges, 2018).

Estima-se que a intervenção ocorria por 40 horas semanais: 10 horas com o estagiário, na residência da criança, em atividades estruturadas; 10 horas com os pais e a babá em atividades de autocuidados, manutenção e generalização das habilidades aprendidas; 20 horas na escola, com direcionamento individualizado e sistemático do mediador. Além disso, a criança fazia sessões semanais de fonoaudiologia, terapia ocupacional (integração sensorial) e musicoterapia em consultório, com duração aproximada de uma hora cada. Também fazia acompanhamento psiquiátrico sistemático e uso de medicação (antipsicótico atípico).

É importante ressaltar que, durante os cinco anos de intervenção, ocorreram trocas de estagiários e profissionais do CEI, na residência e na escola; no geral, os estagiários e profissionais permaneceram por um ano ou mais. Nesse período, a criança foi acompanhada por quatro estagiários na residência e cinco na escola (em momentos diferentes); na residência, foram quatro profissionais de Psicologia e dois de Terapia Ocupacional; na escola, foram quatro profissionais de Psicologia.

\subsection{Programas de ensino}

A Tabela 1, mais adiante, apresenta a sequência de introdução dos programas de ensino e os meses de realização de cada um deles, entre maio de 2012 e maio de 2017. Esses programas foram realizados na residência da criança, por meio da capacitação do estagiário. Ao todo foram realizados 96 programas de ensino: 13 no primeiro ano, 27 no segundo, 19 no terceiro, 13 no quarto e 24 no quinto.

Os procedimentos de ensino das habilidades trabalhadas em cada programa eram fundamentados em análise do comportamento e, para cada programa, eram definidos os critérios para início e término. Após o término de cada programa, eram planejadas estratégias para manutenção e generalização das habilidades aprendidas, preferencialmente em ambiente natural. A descrição detalhada dos programas de ensino de habilidades básicas encontra-se em Gomes e Silveira (2016), de modo a favorecer sua replicabilidade. Informaçóes a respeito dos procedimentos de ensino de habilidades de leitura e matemática podem ser encontrados em Gomes (2007, 2015). 


\subsection{CONSIDERAÇÓES ÉTICAS E FIDEDIGNIDADE}

Os procedimentos empregados, neste estudo, foram aprovados pelo Comitê de Ética da Faculdade de Ciências Médicas de Minas Gerais (Parecer n 923.913) para um projeto maior de investigação sobre capacitação de cuidadores para a Intervençấo Comportamental Intensiva.

Dois avaliadores independentes e não informados quanto ao propósito da pesquisa contabilizaram avaliaçóes (realizadas pelos profissionais do CEI) e 30\% dos protocolos de registro das atividades (preenchidos pelos cuidadores). Fez-se o cálculo de concordância entre observadores, usando-se a fórmula: concordâncias divididas pela somatória de concordâncias e discordâncias, multiplicado por 100 (Kazdin,1982). O coeficiente de concordância para as avaliaçóes foi de $100 \%$; o coeficiente de concordância para os registros das atividades foi de aproximadamente $80 \%$.

\begin{tabular}{|c|c|c|c|c|c|c|c|c|c|c|c|c|c|c|c|}
\hline Ano & Áreas & Programas & Mai & Jun & Jul & Ago & Set & Out & Nov & Dez & Jan & Fev & Mar & Abr & Mai \\
\hline \multirow{13}{*}{ 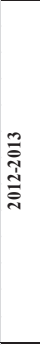 } & \multirow{2}{*}{ Atenção } & Contato visual: 1 segundo & & & & & & & & & & & & & \\
\hline & & Contato visual: à distância & & & & & & & & & & & & & \\
\hline & \multirow{3}{*}{ Imitação } & Movimentos motores grossos & & & & & & & & & & & & & \\
\hline & & Ações com objetos & & & & & & & & & & & & & \\
\hline & & Movimentos fonoarticulatórios & & & & & & & & & & & & & \\
\hline & \multirow{2}{*}{ Linguagem receptiva } & Seguir instruções de um passo & & & & & & & & & & & & & \\
\hline & & Identificação de objetos & & & & & & & & & & & & & \\
\hline & \multirow{3}{*}{ Linguagem expressiva } & Comunicação alternativa: fala sinalizada & & & & & & & & & & & & & \\
\hline & & Pedidos: dá & & & & & & & & & & & & & \\
\hline & & Produzir sons com função comunicativa & & & & & & & & & & & & & \\
\hline & \multirow{3}{*}{ Pré-acadêmicas } & Recortar & & & & & & & & & & & & & \\
\hline & & Emparelhar objetos & & & & & & & & & & & & & \\
\hline & & Colorir & & & & & & & & & & & & & \\
\hline \multirow{27}{*}{ 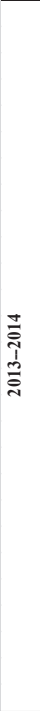 } & \multirow{4}{*}{ Atenção } & Contato visual: 1 segundo & & & & & & & & & & & & & \\
\hline & & Contato visual: 3 segundos & & & & & & & & & & & & & \\
\hline & & Contato visual: 5 segundos & & & & & & & & & & & & & \\
\hline & & Contato visual: ao brincar & & & & & & & & & & & & & \\
\hline & \multirow{6}{*}{ Imitação } & Movimentos motores grossos & & & & & & & & & & & & & \\
\hline & & Ações com objetos & & & & & & & & & & & & & \\
\hline & & Movimentos motores finos & & & & & & & & & & & & & \\
\hline & & Movimentos fonoarticulatórios & & & & & & & & & & & & & \\
\hline & & Movimentos grossos empé & & & & & & & & & & & & & \\
\hline & & Sequência de movimentos & & & & & & & & & & & & & \\
\hline & \multirow{4}{*}{ Linguagem receptiva } & Seguir instruções de um passo & & & & & & & & & & & & & \\
\hline & & Identificação de partes do corpo & & & & & & & & & & & & & \\
\hline & & Identificação de objetos & & & & & & & & & & & & & \\
\hline & & Identificação de figuras & & & & & & & & & & & & & \\
\hline & \multirow{6}{*}{ Linguagem expressiva } & Comunicação alternativa: fala sinalizada & & & & & & & & & & & & & \\
\hline & & Produzir sons com função comunicativa & & & & & & & & & & & & & \\
\hline & & Apontar para itens desejados & & & & & & & & & & & & & \\
\hline & & Imitar sons & & & & & & & & & & & & & \\
\hline & & Nomear pessoas & & & & & & & & & & & & & \\
\hline & & Nomear figuras & & & & & & & & & & & & & \\
\hline & \multirow{7}{*}{ Pré-acadêmicas } & Recortar & & & & & & & & & & & & & \\
\hline & & Emparelhar objetos & & & & & & & & & & & & & \\
\hline & & Emparelhar figuras e objetos & & & & & & & & & & & & & \\
\hline & & Nomear vogais & & & & & & & & & & & & & \\
\hline & & Cópia de letras & & & & & & & & & & & & & \\
\hline & & Contar 1 a 10 & & & & & & & & & & & & & \\
\hline & & Nomear números & & & & & & & & & & & & & \\
\hline \multirow{15}{*}{ 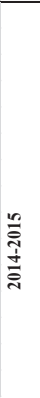 } & & Contato visual: ao brincar & & & & & & & & & & & & & \\
\hline & Atenção & Contato visual: ao brincar e à distância & & & & & & & & & & & & & \\
\hline & & Contato visual: mais de uma pessoa & & & & & & & & & & & & & \\
\hline & Imitação & Sequência de movimentos & & & & & & & & & & & & & \\
\hline & & Identificação de partes do corpo & & & & & & & & & & & & & \\
\hline & Linguagem receptiva & Seguir instruções de dois passos & & & & & & & & & & & & & \\
\hline & & Identificação de objetos & & & & & & & & & & & & & \\
\hline & & Nomeação de pessoas & & & & & & & & & & & & & \\
\hline & Linguagem expressiva & Nomeação de figuras & & & & & & & & & & & & & \\
\hline & & Nomeação de objetos & & & & & & & & & & & & & \\
\hline & & Recortar & & & & & & & & & & & & & \\
\hline & & Nomear vogais & & & & & & & & & & & & & \\
\hline & & Nomear encontros vocálicos & & & & & & & & & & & & & \\
\hline & & Cópia de letras & & & & & & & & & & & & & \\
\hline & Pré-acadêmicas & Contar 1 a 10 & & & & & & & & & & & & & \\
\hline
\end{tabular}




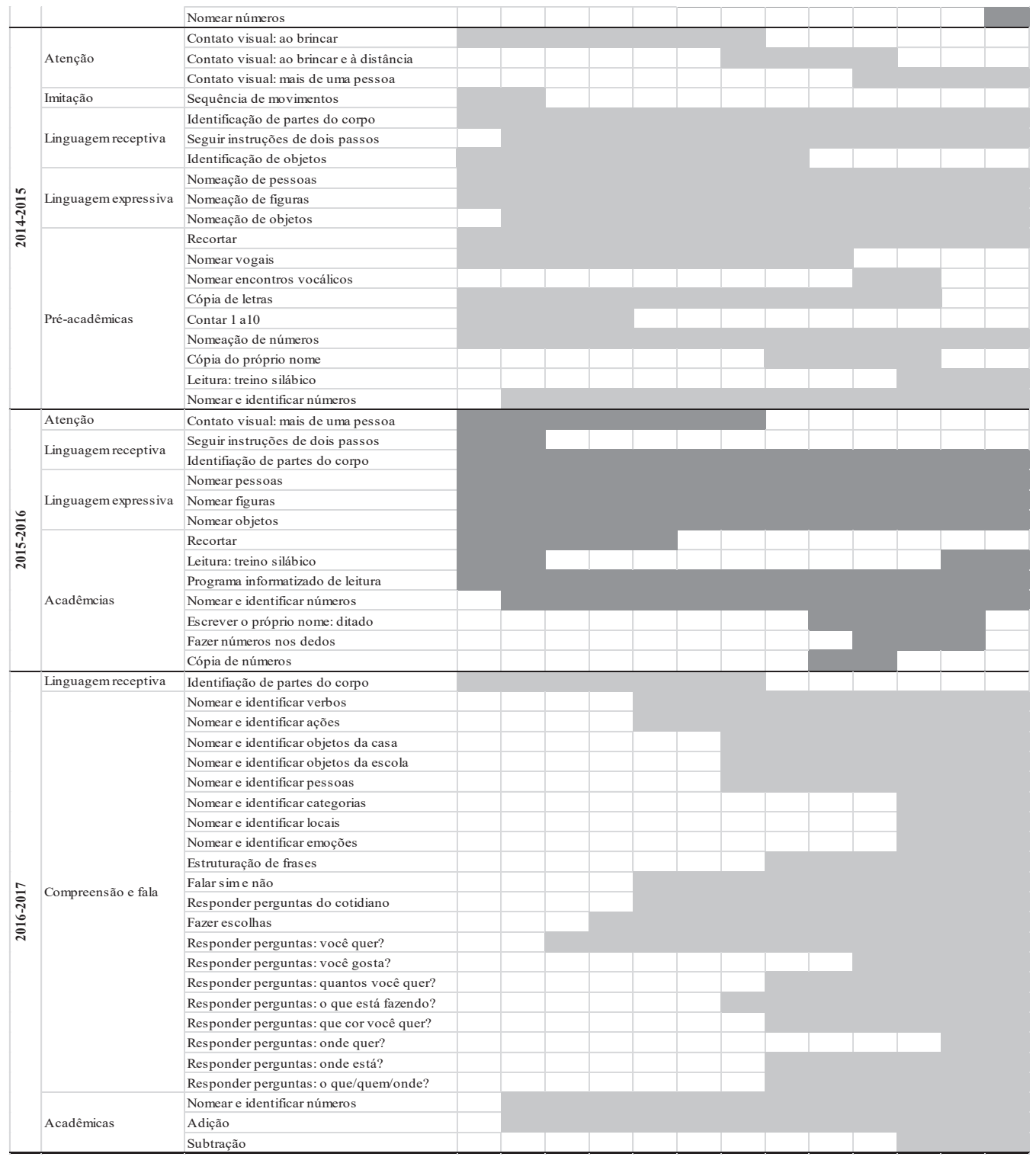

Tabela 1. Sequência de introduçáo dos programas de ensino e os meses de realização de cada um deles, entre maio de 2012 e maio de 2017.

\section{Resultados}

O participante foi avaliado anualmente por meio do PEP-R, IPO e CARS. As avaliações foram aplicadas com intervalo entre elas de aproximadamente 12 meses. O PEP-R foi aplicado seis vezes e o IPO quatro vezes, em funçáo do limite de idade definido pelo instrumento (0 a 6 anos). A CARS foi aplicada 5 vezes, também considerando o critério de idade (acima dos 36 meses). 
As Figuras 1 e 2, ao final desta seção, apresentam os resultados do participante por idade no PEP-R e no IPO, por área do desenvolvimento e na somatória de todas as áreas (Global). Os dois instrumentos apresentam pontuaçôes específicas para cada idade cronológica; a criança está de acordo com o que é esperado para a idade cronológica quando obtém pontuação compatível com a pontuação determinada para aquela idade; pontuação abaixo da determinada indica atraso no desenvolvimento.

De acordo com o PEP-R, a criança apresentava atraso em todas as áreas e na somatória das áreas antes do início da intervenção (Figura 1). De maneira geral a criança apresentou ganhos em todas as áreas, na maior parte das avaliaçôes. Desempenhos mais baixos foram observados em algumas áreas na avaliação realizada aos 5 anos e 4 meses. Essa avaliação coincidiu com um período no qual a criança estava agitada, com muito problemas de comportamento e muitas estereotipias, em função de um tratamento alternativo realizado pela família, que foi rapidamente interrompido. Em relação aos ganhos por área, observam-se ganhos menores em cognitivo e cognitivo verbal; ganhos mais expressivos nessas áreas ocorreram após o aparecimento da fala, entre 4 e 5 anos de idade.

No IPO, a criança apresentava atraso na maioria das áreas, exceto em desenvolvimento motor, antes do início da intervenção (Figura 2). Observam-se ganhos gradativos em todas as áreas, à medida que a intervenção acontece. Desempenhos mais baixos foram observados em linguagem; ganhos mais robustos nessa área foram observados após o aparecimento da fala, entre 4 e 5 anos de idade.

Na primeira avaliação, realizada por meio da CARS, aos 3 anos e 3 meses, a criança apresentou pontuação 40,5 caracterizada como autismo grave. A partir da segunda avaliação, a criança apresentou pontuação compatível com a categoria de autismo leve/moderado: 33 aos 4 anos e 3 meses; 30 aos 5 anos e 4 meses; 34,5 aos 6 anos e 5 meses; e 33 aos 7 anos e 4 meses.
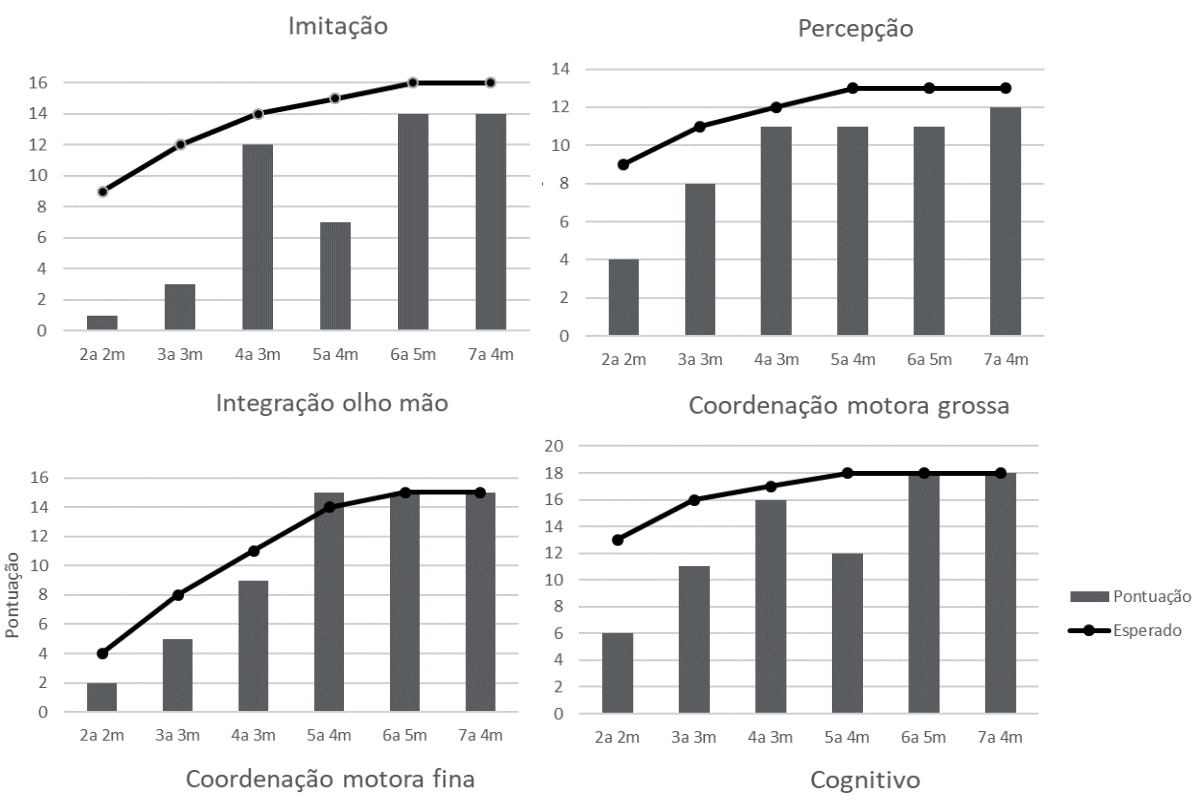


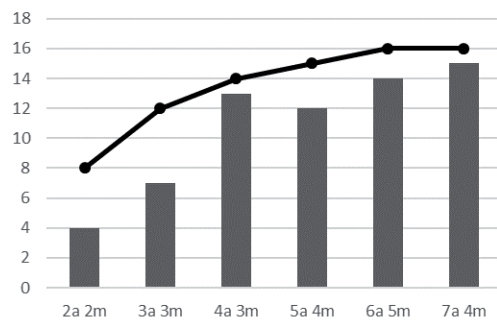

Cognitivo-verbal

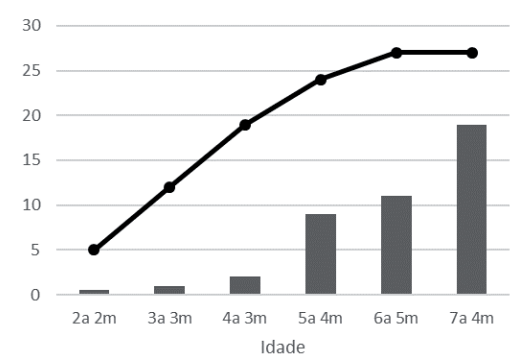

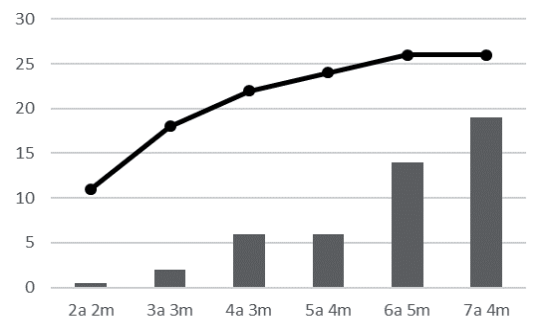

Global

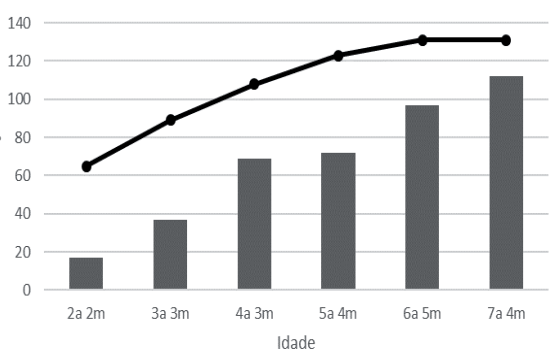

Figura 1. Resultados anuais por área do desenvolvimento e na somatória de todas as áreas, de acordo com o PEP-R. A linha indica pontuação esperada para a idade cronológica e as barras indicam o resultado do participante.

Autocuidados

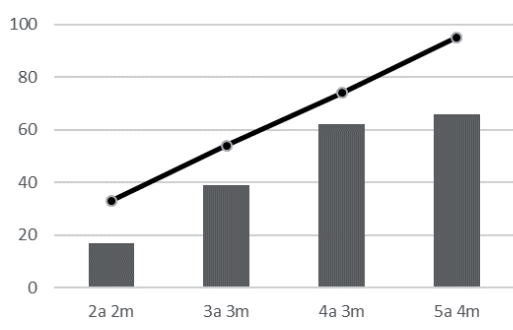

Desenvolvimento motor

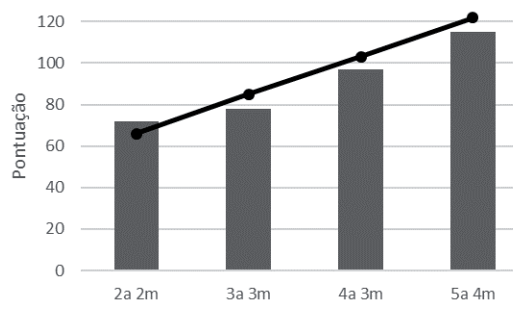

Cognição

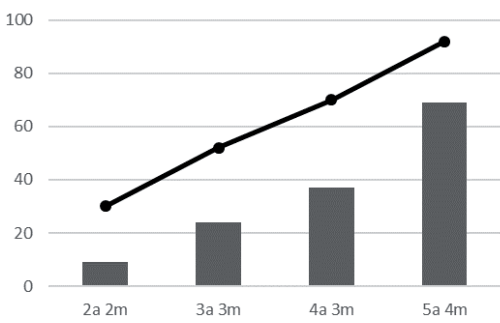

Linguagem

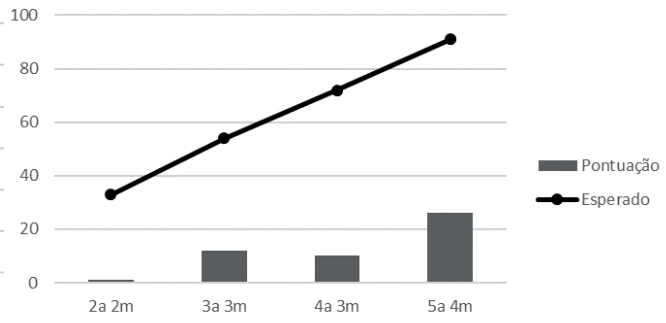



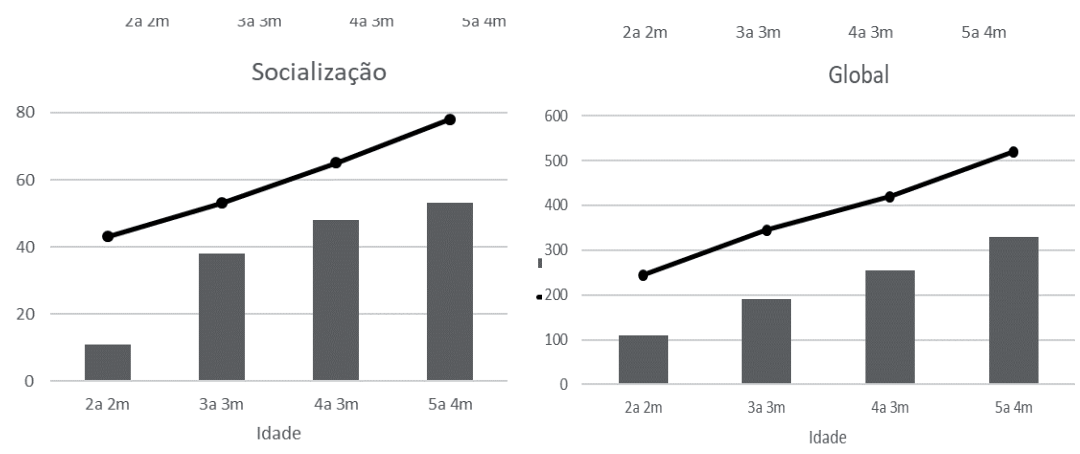

Figura 2. Resultados anuais por área do desenvolvimento e na somatória de todas as áreas, de acordo com o IPO. A linha indica a pontuação esperada para a idade cronológica e as barras indicam o resultado do participante.

\section{Discussão E CONCLUSÓES}

O objetivo deste estudo de caso único foi descrever um modelo de Intervenção Comportamental Intensiva, realizado por meio da capacitaçáo de cuidadores variados, com uma criança com autismo que inicialmente era gravemente comprometida e não falava. Além disso, o estudo pretendeu avaliar os efeitos desse tipo de intervenção, realizada por 40 horas semanais em ambiente domiciliar e escolar, no desenvolvimento da criança, ao longo de cinco anos de intervenção. Dois instrumentos padronizados (PEP-R e IPO) foram utilizados anualmente para medir o desenvolvimento da criança, permitindo avaliar possíveis ganhos decorrentes da intervenção.

De maneira geral, a criança apresentou ganhos gradativos no desenvolvimento, ano após ano. Ganhos mais rápidos foram observados no PEP-R em habilidades mais básicas, como integração olho-mão, percepção, coordenação motora fina e motora grossa. Ganhos mais expressivos em desempenho cognitivo e cognitivo verbal, que envolvem habilidades mais complexas, começaram a aparecer após o surgimento da fala, entre 4 e 5 anos de idade, que, por sua vez, parece ter tido a melhora das habilidades de imitaçáo como precursora, sugerindo que, para essa criança, o refinamento das habilidades de imitação pode ter favorecido o aparecimento da fala.

Outro aspecto importante é que a melhora das habilidades de imitação aconteceu após a aplicaçấo de muitos programas de ensino de imitação (2013-2014), sugerindo uma possível relação entre a intervenção, a melhora da imitação e o surgimento da fala. Os dados do IPO parecem reforçar as indicaçóes do PEP-R: ganhos mais expressivos em cogniçáo e linguagem ocorreram após o aparecimento da fala. Nesse aspecto, a literatura tem indicado que crianças com autismo que falam apresentam ganhos maiores no desenvolvimento, quando comparadas às crianças que não falam (Green, 1996), semelhante ao que foi observado neste estudo atual ao se comparar os ganhos da criança quando ela náo falava e após o aparecimento da fala. 
A respeito da capacitação de cuidadores, a literatura tem indicado que essa pode ser uma alternativa viável e efetiva para a realização de Intervençóes Comportamentais Intensivas. Segundo Smith et al. (2000), a utilização de profissionais formados para realizar toda a intervenção pode ser inviável em função do número reduzido de profissionais capacitados, em relação à demanda de pessoas com autismo a ser atendida, e o alto custo de execução, em função da necessidade de muitas horas de intervenção semanal. Dessa maneira, a solução encontrada para esses problemas tem sido a capacitaçáo de cuidadores, semelhante ao que foi descrito neste estudo. Nesse contexto, diversos estudos utilizaram cuidadores variados para a realização da intervenção (e.g., Anderson, Avery, DiPietro, Edwards, \& Christian, 1987: pais, psicólogos, fonoaudiólogos e professores de educação especial; Dawson et al., 2010: profissionais e pais; Eikeseth, Smith, Jahar, \& Eldevik, 2002: professores; Lovaas, 1987: profissionais e estagiários; Sheinkopf \& Sielgel, 1998: pais; Sherman, Barker, Lorimer, Swinson, \& Factor, 1988: pais e profissionais; Smith et al., 2000: pais e estagiários).

Outro aspecto que merece destaque é a quantidade de anos em que a intervenção foi realizada com essa criança. A literatura indica que Intervençôes Comportamentais Intensivas devem acontecer por pelos menos dois anos consecutivos ou mais (Dawson \& Osterling, 1997; Dawson et al., 2010; Smith et al., 2000; Lovaas, 1987). Pode-se observar que a criança deste estudo apresentou ganhos gradativos com a intervenção, porém ganhos mais robustos nas áreas de linguagem e cognição (ver cognitivo verbal e cognitivo no PEP-R e linguagem e cognição no IPO, Figuras 1 e 2) só aconteceram a partir do terceiro ano consecutivo de intervenção. Antes disso, os ganhos são mínimos nessas áreas, que, por sua vez, são compostas por comportamentos complexos e que demandam tempo para serem aprendidos. Nesse sentido, os dados deste estudo podem sugerir, por um lado, que, para os quadros de autismo mais graves, caracterizados por atraso expressivo no desenvolvimento, ausência de fala e pontuaçáo alta na CARS, como era o perfil do participante deste estudo, a estimulação deve ser ampla (muitas horas semanais) e longa (muitos anos consecutivos), ocorrendo por mais de dois ou três anos consecutivos. Por outro lado, os dados podem sugerir também que a interrupção da intervenção no primeiro ou segundo ano de estimulação poderia comprometer o aparecimento da fala e a aprendizagem de comportamentos mais complexos, o que consequentemente aumentaria o atraso no desenvolvimento da criança. Contudo, trata-se apenas de uma hipótese, pois a falta de um controle experimental mais robusto neste estudo não permite uma afirmação precisa a respeito desse aspecto.

A literatura indica, de maneira consistente, que Intervençóes Comportamentais Intensivas podem promover ganhos significativos no desenvolvimento de crianças com autismo, embora a descrição da proporção dos ganhos seja variada (Lovaas, 1987; Sherman et al., 1988; Smith, 1999; Warren et al., 2011). Os resultados descritos neste estudo corroboram com as indicações da literatura a respeito dos bons efeitos desse tipo de intervenção com crianças com autismo, pois as intervençóes realizadas produziram efeitos expressivos no desenvolvimento do participante. Entretanto, em função de não haver um delineamento experimental robusto para controle das variáveis, o estudo mostra-se limitado em relação à generalização dos dados para outras crianças com autismo, por apresentar apenas um participante, além de problemas no controle de variáveis que podem ter influenciado nos resultados, como a própria maturação da criança e outros tratamentos como variáveis intervenientes. Seria importante que o estudo 
fosse replicado com um número maior de participantes, com um controle melhor das variáveis, além da utilização de um delineamento mais adequado, como no caso do delineamento de linha de base múltipla (Gast, Skouge, \& Tawney, 1984), que possibilitaria acompanhar todo o processo de aprendizagem, antes que as habilidades fossem ensinadas, durante e após o ensino.

\section{REFERÊNCIAS}

Anderson, S. R., Avery, D. L., DiPietro, E. K., Edwards, G. L., \& Christian, W. P. (1987). Intensive home-based early intervention with autistic children. Education and Treatment of Children, 10, 352-366.

Baer, D. M., Wolf, M. M., \& Risley, T. R. (1987). Some still-current dimensions of applied behavior analysis. Journal of Applied Behavior Analysis, 20(4), 91-97.

Boyd, R. D., \& Corley, M. J. (2001). Outcome survey of early intensive behavioral intervention for young children with autism in a community setting. Autism, 5(4), 430-441.

Campbell, M., Schopler, E., Cueva, J., \& Hallin, A. (1996). Treatment of autistic disorder. Journal of the American Academy of Child \& Adolescent Psychiatry, 35(2), 134-143.

Dawson, G., \& Osterling, J. (1997). Early intervention in autism: Effectiveness and common elements of current approaches. In M. J. Guralnick (Ed.), The effectiveness of early intervention: Second generation research (pp. 307-326). Baltimore: Brookes.

Dawson, G., Rogers, S., Munson, J., Smith, M., Winter, J., Greenson, J. ... Varley, J. (2010). Randomized, controlled trial of an intervention for toddlers with autism: The Early Start Denver Model. Pediatrics, 125(1), 17-23.

Eikeseth, S., Smith, T., Jahr, E., \& Eldevik, S. (2002). Intensive behavioral treatment at school for 4-to 7-year-old children with autism: A 1-year comparison controlled study. Behavior Modification, 26(1), 49-68.

Gast, D. L., Skouge, J. R., \& Tawney, J. W. (1984). Multiple baseline designs. Single subject research in special education, 226-268.

Gomes, C. G. S. (2007). Autismo e ensino de habilidades acadêmicas: adição e subtração. Revista Brasileira de Educação Especial, 13(3), 345-364.

Gomes, C. G. S. (2015). Ensino de leitura para pessoas com autismo. Curitiba: Appris.

Gomes, C. G. S., \& Silveira, A. D. (2016). Ensino de habilidades básicas para pessoas com autismo: Manual para Intervenção Comportamental Intensiva. Curitiba: Appris.

Gomes, C., de Souza, D., Silveira, A., \& Oliveira, I. (2017). Intervenção Comportamental Precoce e Intensiva com crianças com Autismo por meio da capacitação de cuidadores. Revista Brasileira de Educação Especial, 23(3), 377-390.

Gomes, C. G. S, Souza, D. das G. de, Silveira, A. D., Rates, A. C, Paiva, G. C de C., \& Castro, N. P. de. (2019). Efeitos da Intervenção Comportamental Intensiva realizada por meio da capacitação de cuidadores de crianças com autismo. Psicologia: Teoria e Pesquisa, 35, e3523.

Gonzaga, M., \& Borges, A. (2018). Tipos de situação de inclusão: uma nova proposta de adaptação curricular para crianças com TEA. In A. Borges, \& M. Nogueira (Eds.). Toda criança pode aprender: o aluno com autismo na escola ( $1^{\text {a }}$ ed., pp. 163-186). Campinas: Mercado de Letras. 
Green, G. (1996). Early behavioral intervention for autism: What does research tell us? In C. Maurice, G. Green, \& S. Luce (Eds.). Behavioral intervention for young children with autism: A manual for parents and professionals (pp. 29-44). Austin (TX): Pro-Ed.

Kazdin, A. E. (1982). Single-case research designs: Methods for clinical and applied settings. New York: Oxford University Press.

Leon, V. C., Bosa, C. A., Hugo, C. N., \& Hutz, C. (2004). Propriedades psicométricas do Perfil Psicoeducacional Revisado: PEP-R. Avaliação Psicológica, 3(1), 39-52.

Lovaas, O. I. (1987). Behavioral treatment and normal educational and intellectual functioning in young autistic children. Journal of Consulting and Clinical Psychology, 55(1), 3-9.

Organização Mundial de Saúde (1993). Classificação de transtornos mentais e de comportamentos da CID10: descriçôes clínicas e diretrizes diagnósticas. Porto Alegre: Artes Médicas.

Pereira, A., Riesgo, R. S., \& Wagner, M. B. (2008). Autismo infantil: tradução e validação da Childhood Autism Rating Scale para uso no Brasil. Jornal de Pediatria, 84(6), 487-494.

Schopler, E., Reichler, R. J., Bashford, A., Lansing, M. D., \& Marcus, L. M. (1990). Individualized assessment and treatment for autistic and developmentally disabled children: Psychoeducational ProfileRevised (PEP-R). Austin (TX): Pro- Ed.

Schopler, E., Reichler, J. R., \& Renner, C. (1988). CARS-The Childhood Autism Rating Scale. Los Angeles: Westerm Pychological Services.

Sheinkopf, S. J., \& Siegel, B. (1998). Home-based behavioral treatment of young children with autism. Journal of Autism and Developmental Disorders, 28(1), 15-23.

Sherman, J., Barker, P., Lorimer, P., Swinson, R., \& Factor, D. C. (1988). Treatment of autistic children: Relative effectiveness of residential, out-patient and home-based interventions. Child Psychiatry and Human Development, 19(2), 109-125.

Silveira, A. D., \& Gomes, C. G. (2019). Ensino de habilidades de autocuidados para pessoas com autismo: Manual para Intervenção Comportamental Intensiva. Belo Horizonte: CEI.

Silveira, A., \& Oliveira, A. (2018). Ensino de habilidades de autocuidado para crianças com autismo em ambiente escolar. In A. Borges, \& M. Nogueira (Eds.). Toda criança pode aprender: o aluno com autismo na escola ( $1^{\mathrm{a}}$ ed., pp. 209-226). Campinas: Mercado de Letras.

Smith, T. (1999). Outcome of early intervention for children with autism. Clinical Psychology: Science and Practice, 6(1), 33-49.

Smith, T., Buch, G. A., \& Gamby, T. E. (2000). Parent-directed, intensive early intervention for children with pervasive developmental disorder. Research in Developmental Disabilities, 21(4), 297-309.

Warren, Z., McPheeters, M. L., Sathe, N., Foss-Feig, J. H., Glasser, A., \& Veenstra-VanderWeele, J. (2011). A systematic review of early intensive intervention for autism spectrum disorders. Pediatrics, 127(5), 1303-1311.

Willians, L. A., \& Aiello, A. L. R. (2001). Inventário Portage operacionalizado. São Paulo: Mennon.

Submetido em 24/03/2019

Reformulado em 14/05/2019

Aceito em 21/05/2019 
ANDALÉCIO, A.C.G.S.A.M. et al. 